\title{
Role of vitrectomy in Staphylococcus epidermidis endophthalmitis
}

\author{
LINDA A FICKER, TRAVIS A MEREDITH, LOUIS A WILSON, \\ AND HENRY J KAPLAN
}

From Emory University School of Medicine, Department of Ophthalmology, Atlanta, Georgia, USA

SUMMARY Seventeen patients with endophthalmitis due to coagulase-negative staphylococcus were treated over a nine-year period with vitrectomy, intraocular antibiotics, and systemic steroids and antibiotics. Fifteen patients presented with moderate to severe disease and visual acuities from counting fingers to light perception, while two had acuities of 20/60 and 20/200. A final visual outcome of $20 / 70$ or better was achieved in 13 of 17 eyes $(76 \%)$. Only one eye lost perception of light secondary to retinal detachment. Therapy including vitrectomy is an effective means of controlling moderate to severe coagulase-negative staphylococcal endophthalmitis and restoring vision.

Coagulase-negative staphylococci are increasingly recognised as pathogenic in postoperative and posttraumatic endophthalmitis. ${ }^{1-7}$ Most of these organisms are probably Staphylococcus epidermidis, but the classification of these organisms is becoming more sophisticated and it is recognised that coagulase-negative Gram-positive micrococci besides Staph. epidermidis may be pathogenic. ${ }^{3}$ In the past, however, these were classified in the group Staph. epidermidis. In general the results of therapy are better when Staph. epidermidis is the infecting organism than when more virulent organisms, especially Gram-negative, are the offending agents. Because virulence may vary within a classification of coagulase-negative organisms, however, general conclusions regarding therapy may be difficult to reach.

O'Day et al. presented good results (20/70 in $83 \%$ ) for management of 18 cases of Staph. epidermidis endophthalmitis with topical and systemic antibiotics and steroids, contrasting these with the less favourable results in cases treated by vitrectomy. ${ }^{2}$ Bode et al. reported a series of 22 eyes treated with intraocular antibiotics; 13 had also undergone vitrectomy. The success rate was $73 \%$ achieving vision of $20 / 60$ or better. ${ }^{5}$ We report a consecutive series of 17 eyes treated over a nine-year period by vitrectomy, intraocular antibiotics, and systemic steroids and antibiotics.

Correspondence to Linda A Ficker, FRCS, Professorial Unit, Moorfields Eye Hospital, City Road, London EC1V 2PD.

\section{Patients and methods}

During the nine years from 1976 to 1985,17 patients were treated at the Emory Clinic for exogenous endophthalmitis due to coagulase-negative staphylococci with therapy which included vitrectomy. Fourteen had undergone prior cataract extraction. Three had postoperative complications (two vitreous wicks and one conjunctival bleb), and one underwent a surgical posterior capsulotomy. Penetrating trauma preceded the infection in two eyes and one case occurred in the postoperative period after trabeculectomy. The onset of the disease 18 months after surgery indicates this was bleb-associated endophthalmitis. In the remaining 16 eyes the onset of symptoms ranged from two to 21 days with a mean of 16.5 days (Table 1 ). Symptoms were initially mild in half the cases (minor discomfort with blurred vision) with transition to a more acute course characterised by pain and sudden visual deterioration 24 hours to 21 days later. Six patients with milder disease initially responded to topical steroid administration, delaying the diagnosis of endophthalmitis. Patients presenting after 48 hours of symptoms $(n=11)$ tended to have more vitreous clouding and involvement of the posterior vitreous.

Preoperative visual acuities ranged from counting fingers to light perception in 15 cases; the remaining two were $20 / 60$ and $20 / 200$. Vitreous surgery was undertaken promptly after diagnosis. Indications for surgery were moderate to severe intraocular inflam- 
Table 1 Clinical details summarised

\begin{tabular}{|c|c|c|c|c|c|c|}
\hline Case & Event & $\begin{array}{l}\text { Days from Event } \\
\text { to symptoms }\end{array}$ & $\begin{array}{l}\text { Days from Symptoms } \\
\text { to diagnosis }\end{array}$ & Sequelae & Final $V A$ & $\begin{array}{l}\text { Followup } \\
\text { (post-vitrectomy) }\end{array}$ \\
\hline 1 & ICCE & 7 & 2 & - & $20 / 30$ & 7 years \\
\hline 2 & ICCE & 7 & 42 & - & $20 / 70$ & 6 months \\
\hline 3 & ICCE & 3 & 3 & - & $20 / 30$ & 2 years \\
\hline 4 & ICCE & 5 & 1 & - & $20 / 30$ & $4 \cdot 5$ years \\
\hline 5 & ICCE & 4 & 1 & 一 & $20 / 70$ & 6 years \\
\hline 6 & ICCE Vitreous wick & 21 & 5 & CMO & $20 / 400$ & 7 years \\
\hline 7 & ICCE & 5 & 1 & - & $20 / 30$ & 5 years \\
\hline 8 & ICCE Vitreous wick & 7 & 1 & RD & NPL & \\
\hline 9 & ECCE/IOL & 5 & 42 & 一 & $20 / 60$ & 15 years \\
\hline 10 & ECCE/IOL & 2 & 1 & RD/ABK & CF & 6 months \\
\hline 11 & ECCE/IOL & 2 & 4 & RD & $20 / 30$ & 6 months \\
\hline 12 & $\mathrm{ECCE} / \mathrm{IOL}$ & 3 & 10 & - & $20 / 60$ & 21 months \\
\hline 13 & $2^{\prime}$ IOL Bleb & 540 & 14 & - & $20 / 60$ & 4 years \\
\hline 14 & Capsulotomy & 2 & 3 & - & $20 / 60$ & $4 \cdot 5$ years \\
\hline 15 & Trabeculectomy & 4 & 6 & - & $20 / 200$ & 5 years \\
\hline 16 & Penetrating trauma & 7 & 4 & - & $20 / 25$ & 21 months \\
\hline 17 & Penetrating trauma & 4 & 3 & - & $20 / 50$ & 9 months \\
\hline
\end{tabular}

'Event' signifies the surgery or trauma preceding endophthalmitis. ICCE: intracapsular cataract extraction. ECCE: extracapsular cataract extraction. IOL: intraocular lens. 2' IOL: secondary intraocular lens. CMO: cystoid macular oedema. RD: retinal detachment. ABK: aphakic bullous keratopathy. NPL: no perception of light.

mation with loss of fundus detail on indirect ophthalmoscopy ( 15 cases), diagnostic difficulty secondary to delayed onset (1), and trauma-associated endophthalmitis (1).

Biopsy material obtained under sterile conditions was immediately inoculated on standard microbiological media and microscopic slides were prepared. Vitrectomy was performed through an anterior limbal approach in nine cases, while eight were operated on by the pars plana. At the conclusion of surgery intraocular antibiotics were injected as follows: gentamicin 100 to $400 \mu \mathrm{g}$ plus a cephalosporin or loridine $100 \mu \mathrm{g}$ or methicillin $250 \mu \mathrm{g}$. Highdose prednisolone $(60 \mathrm{mg})$ was started 24 hours postoperatively in most cases (15/17) and rapidly tailed. The postoperative regimen included an intravenous cephalosporin or ticarcillin plus topical sixhourly gentamicin $(14 \mathrm{mg} / \mathrm{ml})$ and two-hourly prednisolone acetate $1 \%$ in most cases.

\section{Results}

Visual results were gratifying, with $20 / 70$ or better achieved in 13 of the $17(76 \%)$ patients. In the cases with vision less than $20 / 70$, one was due to preexisting end-stage glaucoma. Aphakic bullous keratopathy followed retinal detachment in one case, and cystoid macular oedema was present in another, possibly associated with the preoperative vitreous wick. A single case had an inoperable retinal detachment and vision was totally lost (Table 1).

The major complication in these cases was the development of retinal detachment $(18 \%)$. One detachment occurred in an eye with a vitreous wick and a cyclitic membrane at the time of vitrectomy. Surgical repair was unsuccessful. The remaining two cases were due to presumed entry-site complications. They were successfully repaired, though one had postoperative bullous keratopathy which limited postoperative vision to counting fingers. In this series antibiotic sensitivities of the pathogens cultured were as follows: gentamicin $100 \%$ and cephalothin $83 \%$. Where resistance to cephalothin was reported, the organisms were sensitive to methicillin.

\section{Discussion}

The management of coagulase-negative staphylococcus endophthalmitis remains controversial, since various forms of therapy are reported to be successful. Unfortunately, reported series may not be comparable owing to differences in the virulence of organisms, severity of the disease at presentation, delay in onset, antibiotic sensitivities, and host response. O'Day et al. reported good results from non-invasive therapy, with $83 \%$ of patients achieving vision of $20 / 70$ or better. ${ }^{2}$ They contrasted their results with those reported in patients treated by vitrectomy. Final vision of 20/70 was achieved in only $42 \%$ of these cases. Bode et al. reported 22 eyes treated with intraocular antibiotics; $73 \%$ achieved $20 / 60$ or better. ${ }^{5}$ The condition of these 13 eyes was judged severe, however, and they underwent additional vitrectomy. Vision of 20/60 was achieved in $62 \%$ of this subgroup. Our series demonstrates excellent results in eyes treated with vitrectomy, 
intraocular antibiotics, and systemic steroids and antibiotics. Most eyes in this series had moderate to severe disease and $89 \%$ had a vision of counting fingers or less, with obscuration of all fundus detail at the time of surgical intervention.

Early mild disease may respond well to intraocular antibiotic therapy alone without vitreous removal. When the clinical presentation is that of an indolent endophthalmitis, the clinical picture may strongly suggest Staphylococcus epidermidis, and such cases may be managed initially without surgical intervention. Confronted with acute endophthalmitis, either moderate or severe, or established disease with severe intraocular inflammation, the clinician cannot always be confident he is dealing with an organism of low virulence which is likely to respond to antibiotic therapy alone. If therapeutic vitrectomy is delayed, pending microbiological diagnosis, the delay may allow progression of intraocular inflammation. The potential therapeutic advantage of surgical vitrectomy may thereby be compromised. Immediate vitrectomy, performed at the time of vitreous biopsy in such cases, has three advantages: (1) living organisms are removed, enhancing control of infection; (2) removal of dead organisms reduces the inflammatory stimulus and consequent inflammatory chorioretinal damage which determines visual outcome; (3) removal of vitreous opacities expedites visual recovery.

When a case of moderate to severe bacterial endophthalmitis presents to our clinic, we concur with the recommendations to obtain a vitreous sample quickly in order to establish a microbiological diagnosis. Since needle aspiration may transmit unacceptable traction to the peripheral retina, we usually prefer to remove the vitreous with a suctioncutting instrument. When performed in this way, the biopsy may be extended to remove a larger amount of vitreous. In the presence of complete posterior vitreous detachment this would not result in traction on inflamed, oedematous retina, and most of the vitreous may be removed with relative safety. Where posterior vitreous detachment has not occurred or is incomplete, limited 'core' vitrectomy would minimise the risk of retinal traction while reducing the inflammatory load and vitreous opacification. There would remain the risk of the late effects of subsequent detachment of the residual vitreous cortex.

The major complication of intraocular surgery in this setting is the development of retinal detachment. Sympathetic ophthalmitis has also been reported. ${ }^{8}$ In our series two of the three retinal detachments were successfully repaired. The third detachment could not be repaired and was associated with known preoperative risk factors in the anterior segment, a cyclitic membrane and vitreous wick. Even with these complications, however, only one eye in the series of 17 was ultimately lost, and the final visual results of this series are comparable to reports with antibiotic therapy alone. Vitreous surgery did not compromise the prognosis in these eyes with Staphylococcus epidermidis endophthalmitis and provided useful adjunctive therapy in moderate to severe cases. Although systemic antibiotics were administered, we recognise there is no evidence that minimum inhibitory concentrations are achieved in the vitreous by this route.

The role of steroid therapy is to suppress the massive inflammatory infiltration of the choroid and retina which results from intraocular infection. The inflammatory stimulus includes both live and dead organisms. Hence intraocular antibiotics alone will not contain the inflammatory response. Studies to date have not established the optimum route or dose of steroid administration. O'Day et al. reported the use of predominantly topical or periocular steroid, while our cases were mostly (15 of 17) treated with systemic steroids. ${ }^{2}$ This will need more detailed analysis, relating results to the degree of inflammation and to the different species of coagulasenegative staphylococci.

Staphylococcus epidermidis is widely reported as pathogenic for endophthalmitis. ${ }^{1-7}$ Until recently its identification has been based on the coagulase test. Supplementary tests are required to further differentiate coagulase-negative species of staphylococci, since this may have clinical significance. Staph. haemolyticus, for example, is coagulase-negative but parallels Staph. aureus with respect to virulence and antibiotic sensitivity. More recently Seal et al. ${ }^{4}$ modified the Baird-Parker scheme both to quantify a number of tests and to apply these specifically to coagulase-negative cocci of ophthalmic importance. There is also evidence that slime production may provide a useful index of pathogenicity. ${ }^{9}$ It is important to distinguish between species in order to interpret the results of different types of treatment.

We recommend consideration of vitrectomy in moderate and severe endophthalmitis combined with intraocular antibiotic therapy and systemic steroids. This approach permits adequate vitreous biopsy, reduces the inflammatory stimulus of live and dead bacteria, reduces endotoxin release from more virulent organisms, and clears the visual axis. The results of a surgical approach are not inferior to nonsurgical management as previously suggested.

This work was supported in part by a departmental grant from Research to Prevent Blindness, Inc.

\section{References}

1 Valenton MJ, Brubaker RF, Allen HF. Staphylococcus epider- 
midis (albus) endophthalmitis. Report of two cases after cataract extraction. Arch Ophthalmol 1973; 89: 94-6.

2 O'Day DM, Jones DB, Patrinely J, Elliott JH. Staphylococcus epidermidis endophthalmitis. Visual outcome following noninvasive therapy. Ophthalmology 1982; 89: 354-60.

3 Packer AJ, Koontz FP. Ocular staphylococcal infections. Am J Ophthalmol 1984; $97: 645$.

4 Seal D, Wright P, Badiani D. New ways to classify staphylococci of ophthalmic importance. Invest Ophthalmol Vis Sci 1986; 27 (suppl): 88.

5 Bode DD Jr, Gelender H, Forster RK. A retrospective review of endophthalmitis due to coagulase-negative staphylococci. $\mathrm{Br} \mathrm{J}$ Ophthalmol 1985; 69: 915-9.

6 Puliafito CA, Baker AS, Haaf J, Foster CS. Infectious endo- phthalmitis. Review of 36 cases. Ophthalmology 1982; 89: 921-9.

7 Weber DJ, Hoffman KL, Thoft RA, Baker AS. Endophthalmitis following intraocular lens implantation: report of 30 cases and review of the literature. Rev Infect Dis 1986; 8: 12-20.

8 Croxatto JO, Galantine P, Cupples HP, Harper D, Reader A Zimmerman LE. Sympathetic ophthalmia after pars plana vitrectomy-lensectomy for endogenous bacterial endophthalmitis. Am J Ophthalmol 1981; 91: 342-6.

9 Davenport DS, Massanari RM, Pfaller MA, Bale MJ, Streed SA, Hierholzer WJ Jr. Usefulness of a test for slime production as a marker for clinically significant infections with coagulase-negative staphylococci. J Infect Dis 1986; 153: 332-9.

Accepted for publication 20 March 1987. 